\title{
МЕТОДИКА КОЛИЧЕСТВЕННОЙ ОЦЕНКИ ЗАЩИЩЕННОСТИ ТЕЛЕКОММУНИКАЦИОННЫХ СИСТЕМ
}

В работе приведена методика вычисления количественной оценки защищенности телекоммуникационных систем, позволяющая числено оценить степень соответствия различных защищенных систем выдвигаемым требованиям.

Введение. Наблюдаемое в последнее время прогрессирующее влияние информационных технологий практически на все сферы жизнедеятельности человечества вызывает поступательный рост требований к телекоммуникационным системам, компьютерным сетям и устройствам телекоммуникации. Это объясняется тем, что данные системы являются основным средством обмена информацией и качество их функционирования является определяющим фактором эффективности большинства информационных технологий. Важнейшей составляющей качества функционирования телекоммуникационных систем является качество защиты информации. Обеспечение этой составляющей в настоящее время сталкивается с целым рядом проблем, основной из которых выступает противоречие между потенциальными возможностями существующих подходов и постоянно возрастающими требованиями к защите информации.

Цель работы. Целью работы является дать разработчикам сетей методику, которая позволила бы числено оценить степень соответствия различных защищенных систем выдвигаемым требованиям.

Основная часть. Пусть требуется выбрать одну из нескольких систем для решения конкретной задачи. Предлагаемая методика заключается в следующем: определение набора атрибутов, описывающих системы. Каждый атрибут имеет численную меру - значения; определение значения атрибутов, требуемых решаемой задачей; определения степени соответствия значений атрибутов каждой системы требованиям задачи; определение относительной важности атрибутов с точки зрения решаемой задачи.

Пусть $A=\left[A_{1}, A_{2}, \ldots, A_{n}\right]$ является набором атрибутов, используемый для описания требований задачи и ресурсов системы, примерами которых могут быть производительность, модульность, устойчивость к сбоям и отказам, удобство обслуживания и т.П. Пусть $S=\left[S_{1}, S_{2}, \ldots, S_{N}\right]$ - множество систем, которые потенциально могут быть использованы для решения задач. Каждую из этих систем будем характеризовать значениями атрибутов $A_{1}, \ldots, A_{n}$. Пусть $R=\left[r_{1}, r_{2}, \ldots, r_{n}\right]$ есть значение атрибутов, которые требуются для решения поставленной задачи. $\Theta$ является матрицей $N_{x n}$, которая определяет степень соответствия характеристик рассматриваемых систем требованиям задачи, так что $q_{j i}$ указывает в какой степени значения атрибута $A_{i}$ системы $S_{i}$ соответствует требованию $r_{i}, 1 \leq i \leq n, 1 \leq j \leq N$.

Будем считать, что набор атрибутов $A_{1}, A_{2}, \ldots, A_{n}$ упорядочен в порядке увеличения их важности. Числено относительная важность атрибутов характеризуется вектором весов $\left[W_{1}, W_{2}, \ldots, W_{n}\right]$. Графически введенные понятия иллюстрируются на рис. 1.

В качестве меры соответствия ресурсов системы $S_{l}$ требованиям задачи используется взвешенная разность: $D_{l}=\sum_{i=1}^{n} d_{l i}=\sum_{i=1}^{n}\left(\alpha_{l i}-1\right) W_{i}, \alpha_{l i}=\frac{q_{l i}}{r_{i}}$

Графически $D_{l}$ представлена на рис. 1 суммой площадей заштрихованных прямоугольников. Если $q_{l i}>1$, то можно говорить, что атрибут (ресурс) $i$ имеется в избытке, 
если $q_{l i}>1$, будем говорить о дефиците $i$. Система $S^{*}$ есть оптимальная система из набора $\left[S_{1}, S_{2}, \ldots, S_{N}\right]$ для решения поставленной задачи, если

$$
S^{*}=S_{k}, D_{k}=\max \left\{D_{1}, D_{2}, \ldots, D_{N}\right\}, 1 \leq k \leq N
$$

Выражение (1) сформировано в предположении, что дефицит одного ресурса может быть скомпенсирован избытком другого. Если некоторый ресурс не должен опускаться ниже некоторого уровня (критический дефицит) то выражение (1) необходимо дополнить очевидными неравенствами.

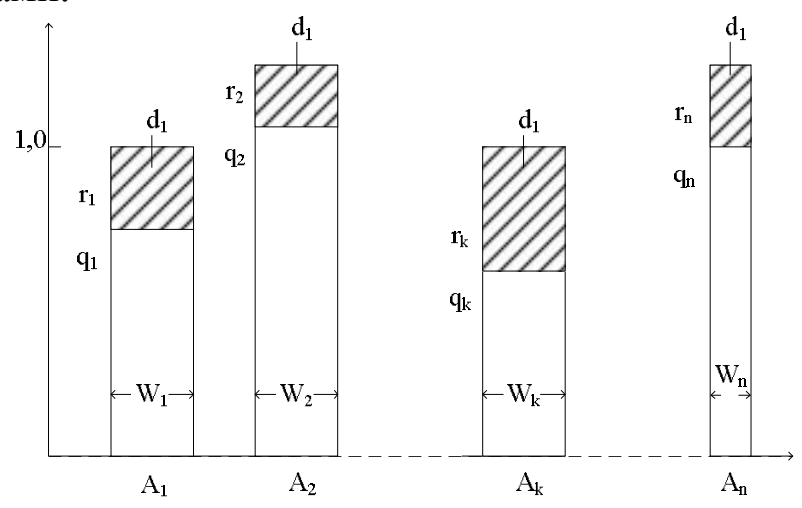

Рис. 1

Теперь осуществим присвоение весов атрибутам. Предположим, весовая функция относится к одному из четырех классов, показанных на рис. 2.

Класс I $W_{i}=1$;

Класс II $W_{i}=1-\left(\frac{i-1}{n-1}\right), 1 \leq i \leq n$;

Класс III $W_{i}=\left\{\begin{array}{l}1,1 \leq i \leq n_{0} \leq n \\ 1-\frac{1-n_{0}}{n-n_{0}}, n_{0} \leq i \leq n\end{array}\right.$;

Класс IV $W_{i}=e^{-c(i-1)}, 1 \leq i \leq n$.

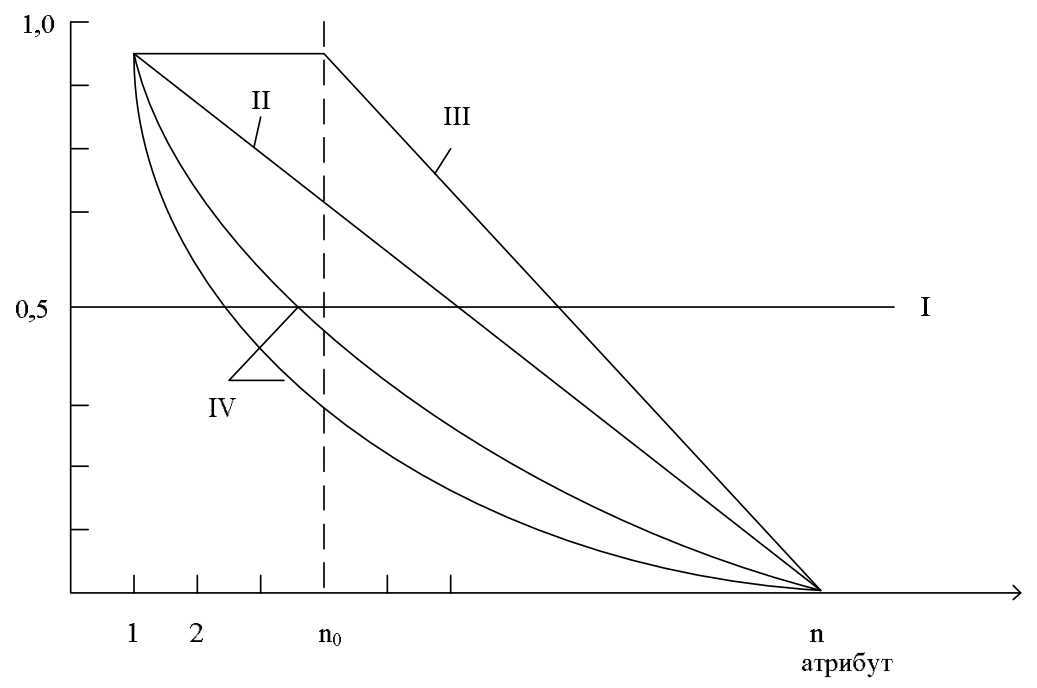

Рис. 2

Взвешенные разности $D_{l}$ для этих классов весовых функций имеет вид

Класс I $D_{l}=\sum_{i=1}^{n} d_{l i}=\sum_{i=1}^{n}\left(\alpha_{l i}-1\right) W_{i}=\sum_{i=1}^{n}\left(\alpha_{l i}-1\right)$. 
Класс II $D_{l}=\sum_{i=1}^{n}\left(\alpha_{l i}-1\right)\left[1-\frac{i-1}{n-1}\right]=\frac{1}{n-1} \sum_{i=1}^{n}\left(\alpha_{l i}-1\right)(n-1)$.

Класс III $D_{l}=\sum_{i=1}^{n_{0}}\left(\alpha_{l i}-1\right)+\sum_{i=n_{0}+1}^{n}\left(\alpha_{l i}-1\right)\left[1-\frac{i-n_{0}}{n-n_{0}}\right]=\sum^{n_{0}}\left(\alpha_{l i}-1\right)+\frac{1}{n-n_{0}} \sum_{i=n_{0}+1}^{n}\left(\alpha_{l i}-1\right)(n-i)$.

Класс IV $D_{l}=\sum_{i=1}^{n}\left(\alpha_{l i}-1\right) e^{-c(i-1)}$.

Взвешенная разность $D_{l}$ есть мера относительная и позволяет только осуществить выбор оптимальной распределенной системы среди потенциально пригодных

Займемся определением числа атрибутов. Будем рассматривать следующие вопросы:

— каково влияние дополнительного атрибута $A_{n+1}$ к набору $\left[A_{1}, A_{2}, \ldots, A_{n}\right]$ ?

- как изменится уровень доверительности при переходе от $n$ атрибутов к $n^{\prime}$, $n^{\prime}>n ?$

— каков эффект декомпозиции некоторого атрибута на набор податрибутов?

Вводится понятие доверительного уровня численной характеристики, применимой как к отдельному атрибуту, так и к набору атрибутов в целом. Пусть $c_{i}$ есть доверительный уровень атрибута $A_{i}$.

$$
c_{i}=\frac{1}{3}\left(c_{i 1}+c_{i 2}+c_{i 3}\right),
$$

где $c_{i 1}$ - представляет степень доверительности в связи с относительной позицией атрибута $A_{i}$ в наборе (его важности); $c_{i 2}$ - представляет степень доверительности оценки параметра $q_{i 1}$; коэффициент $c_{i 3}$ - связан с оценкой параметра $r l_{i}, 0 \leq c_{i} \leq 1,0 \leq c_{i k} \leq 1$, $1 \leq k \leq 3$.

Уровень доверительности из набора из $n$ атрибутов есть:

$$
c^{n}=\frac{1}{n} \sum_{i=1}^{n} c_{i}=\frac{1}{3 n} \sum_{i=1}^{n}\left(c_{i 1}+c_{i 2}+c_{i 3}\right), 0 \leq c^{n} \leq 1 .
$$

Пусть к набору $\left[A_{1}, A_{2}, \ldots, A_{i}, A_{i+1}, \ldots, A_{n}\right]$ добавляется атрибут $A_{n=1}$, который по важности занимает место между $A_{i}$ и $A_{l+1}$ так что набор принимает вид $\left[A_{1}, A_{2}, \ldots, A_{i}, A_{n+1}, A_{i+1}, \ldots, A_{n}\right]$. Естественно допустить, что уровень достоверности, связан с $q_{l i}$ и $r_{i}$ для атрибутов $A_{1}, A_{2}, \ldots, A_{n}$ не изменяется. Уровни доверительности, связанные с относительной важностью изменяются только для атрибутов $A_{i}, A_{i+1}$. Изменение в суммарном уровне доверительности есть:

$$
\begin{aligned}
& c^{n+1}-c^{n}=\frac{1}{3(n+1)}\left[\left(c_{11}+c_{12}+c_{13}\right)+\left(c_{21}+c_{22}+c_{23}\right)+\ldots+\left(c_{j 1}-\delta+c_{j 2}+c_{j 3}\right)+\right. \\
& \left.\left(c_{n+1,1}+c_{n+1,2}+c_{n+1,3}\right)+\left(c_{j+1,1}-\delta+c_{j+1,2}+c_{j+1,3}\right)+\ldots+\left(c_{n 1}+c_{n 2}+c_{n 3}\right)\right]- \\
& -\frac{1}{3 n}\left[\left(c_{11}+c_{12}+c_{13}\right)+\left(c_{21}+c_{22}+c_{23}\right)+\ldots+\left(c_{j 1}+c_{j 2}+c_{j 3}\right)+\left(c_{j+1,1}+c_{j+1,2}+c_{j+1,3}\right)+\right. \\
& \left.+\ldots+\left(c_{n 1}+c_{n 2}+c_{n 3}\right)\right],
\end{aligned}
$$

где $\delta$ - изменение в уровне доверительности атрибутов $A_{j}$ и $A_{j+1}$ в связи с изменениями порядка следования атрибутов. Преобразуя выражения (2), получим 


$$
\begin{aligned}
& c^{n+1}-c^{n}=\left[\frac{1}{3(n+1)}-\frac{1}{3 n} \sum_{i=1}^{n}\left(c_{i 1}+c_{i 2}+c_{i 3}\right)+\frac{1}{3(n+1)}\left(c_{n+1,1}+c_{n+1,2}+c_{n+1,3}\right)-2 \delta\right]= \\
& =\frac{-c^{n}}{n+1}+\frac{1}{3(n+1)}\left[\left(c_{n+1,1}+c_{n+1,2}+c_{n+1,3}\right)-2 \delta\right]=\frac{\left(c_{n+1,1}+c_{n+1,2}+c_{n+1,3}\right)-\left(3 c^{n}+2 \delta\right)}{3(n+1)},
\end{aligned}
$$

графически полученное выражение представлено на рис 3. Отметим, что

$$
0 \leq\left(c_{n+1,1}+c_{n+1,2}+c_{n+1,3}\right) \leq 3 \frac{-(3 c r+2 \delta)}{3(n+1)} \text { и } 0 \leq\left(3 c^{n}+2 \delta\right) \leq 5 \text {. }
$$

Кривые рис. 3 отвечают на вопросы связанные с введением атрибутов. По аналогии с предыдущем рассуждением легко показать, что при разбиении атрибута $A_{k}$ на податрибуты $A_{k 1}, A_{k 2}, \ldots, A_{k m}$ суммарный уровень доверительности изменяется следующим образом:

$$
\begin{aligned}
& (n+m)\left[c^{n+m}-c^{n}\right]=\frac{1}{3}\left[\left(c_{k 11}+c_{k 12}+c_{k 13}\right)+\ldots+\right. \\
& \left.+\left(c_{k m 1}+c_{k m 2}+c_{k m 3}\right)-\left(c_{k 1}+c_{k 2}+c_{k 3}\right)\right]-m c^{n} .
\end{aligned}
$$

Графически полученное выражение представлено на рис. 4.

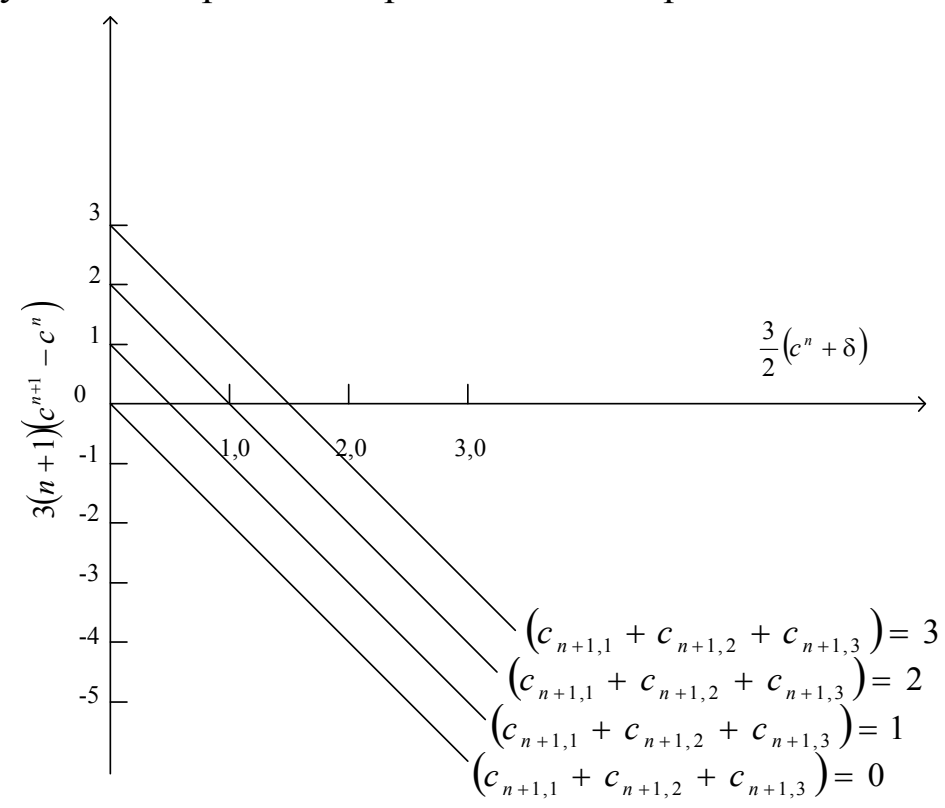

Рис. 3 


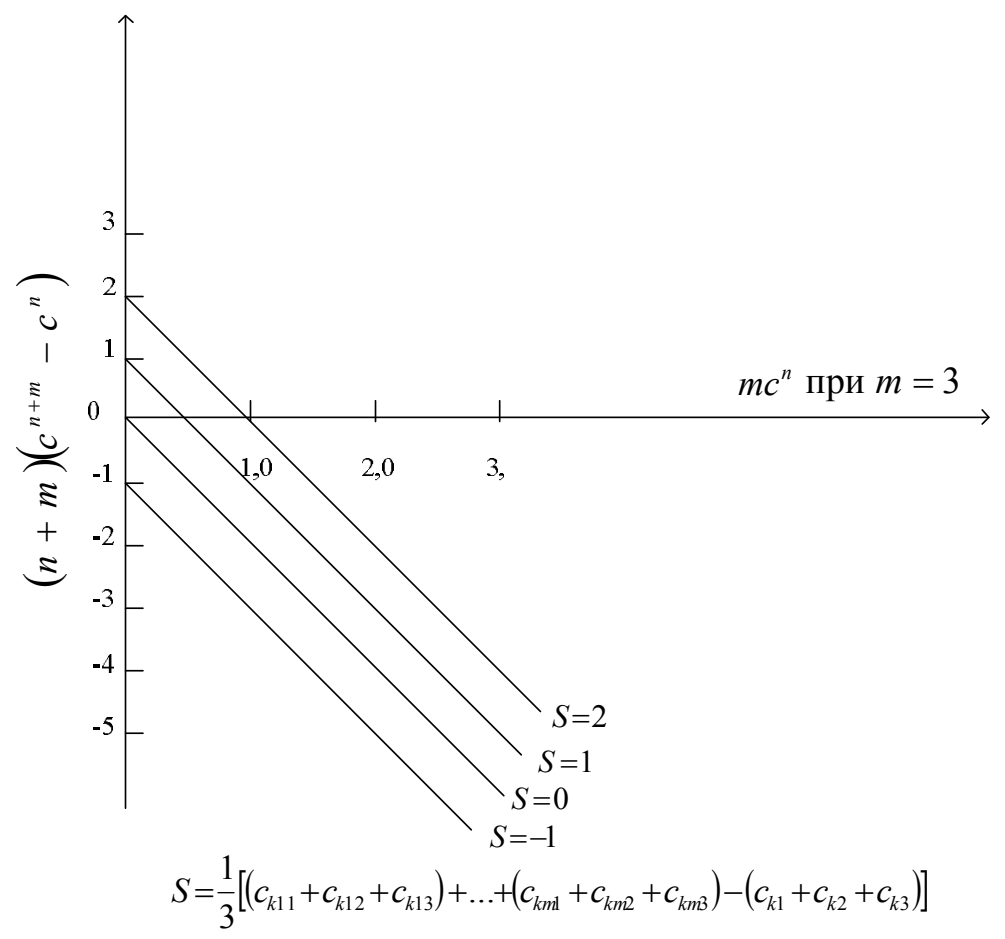

Рис. 4

Выводы. С помощью предложенной методики разработчики сетей могут численно оценить степень соответствия защищенной системы выдвигаемым требованиям, при чем с учетом различных наборов атрибутов с определением их значений и с определением степени соответствия значений атрибутов каждой системы требованиям поставленных задач. А также определение относительной важности атрибутов с точки зрения решаемой задачи. Благодаря этой методике можно выбрать оптимальную систему среди потенциально пригодных систем. В методике также приведены расчеты влияния на систему в целом наличие дополнительных атрибутов с их различной важностью и податрибутов.

Надійшла: 20.09.2011

Рецензент: д.т.н., проф. Хорошко В.О. 